\title{
El desarrollo de la conciencia ambiental en niños de cuatro y cinco años en un colegio prescolar oficial
}

The development of environmental awareness in four- and five-year-old children in an official preschool

0 desenvolvimento da consciência ambiental em crianças de quatro e cinco anos em uma pré-escola oficial

\section{Silvia Marulanda ${ }^{1}$}

Corporación Universitaria Iberoamericana, Colombia

\section{Brenda Millan}

Corporación Universitaria Iberoamericana, Colombia

Luz Sua

Corporación Universitaria Iberoamericana, Colombia

$$
\text { D0I: https://doi.org/10.35622/j.rep.2021.02.001 }
$$

\section{Recibido 02/12/2020/ Aceptado 20/03/2021}

RESUMEN. Dada las situaciones ambientales y la necesidad de su conservación, no solo a mundial sino también a nivel nacional y local, se hace necesaria la búsqueda de apropiación por el cuidado hacia el medio ambiente por parte de niños y niñas desde edad preescolar. Por ello, se diseñó esta investigación con el objetivo de comprender la experiencia de los niños y niñas entre 4 y 5 años frente la responsabilidad ambiental en un colegio oficial de Tuluá, Valle, bajo el paradigma cualitativo, utilizando técnicas de carácter fenomenológico, con una población de 11 estudiantes de preescolar, la selección de la muestra fue no probabilística por conveniencia. Para la obtención de datos se realizó una revisión bibliográfica y se diseñó un guion para las entrevistas. La promoción de la conciencia ambiental en niños y niñas permitió identificar que este proceso se adquiere a través de la interrelación con las demás personas (cuidadores principales y maestros), además del desarrollo de actitudes de identificar actitudes de cuidado y protección del medio ambiente.

PALABRAS CLAVE: responsabilidad ambiental, modelos de aprendizaje, cultura ambiental, conciencia ambiental.

\begin{abstract}
Given the environmental situations and the need for its conservation, not only globally but also nationally and locally, it is necessary to seek appropriation for the care of the environment by children from preschool age. For this reason, this research was designed with the aim of understanding the experience of boys and girls between 4 and 5 years old facing environmental responsibility in an official school in Tuluá, Valle, under the qualitative paradigm, using phenomenological techniques, with a population of 11 preschool students, the selection of the sample was non-probabilistic for convenience. To obtain data, a bibliographic review was carried out and a script for the interviews was designed. The promotion of environmental awareness in children made it possible to identify that this process is acquired through interrelation with other people (main caregivers and teachers), in addition to the development of attitudes to identify attitudes of care and protection of the environment.
\end{abstract}

KEYWORDS: environmental responsibility, learning models, culture awareness, environmental awareness.

\footnotetext{
${ }^{1}$ Correspondencia: silviaromma@hotmail.com
} 
RESUMO. Dadas as situações ambientais e a necessidade de sua conservação, não só global, mas também nacional e localmente, é necessário buscar a apropriação para o cuidado com o meio ambiente pelas crianças desde a idade préescolar. Portanto, esta pesquisa foi elaborada com o objetivo de compreender a vivência de meninos e meninas de 4 a 5 anos frente à responsabilidade ambiental em uma escola oficial de Tuluá, Valle, sob o paradigma qualitativo, por meio de técnicas fenomenológicas, com uma população de 11 pré-escolares, a seleção da amostra foi não probabilística por conveniência. Para a obtenção dos dados, foi realizada uma revisão bibliográfica e elaborado um roteiro para as entrevistas. A promoção da consciência ambiental nas crianças possibilitou identificar que esse processo se adquire por meio da interrelação com outras pessoas (cuidadores principais e professores), além do desenvolvimento de atitudes para identificar atitudes de cuidado e proteção ao meio ambiente.

PALABRAS CLAVE: responsabilidade ambiental, modelos de aprendizagem, cultura ambiental, consciência ambiental.

\section{INTRODUCCIÓN}

Dada las situaciones ambientales y la necesidad de su conservación no solo a nivel mundial sino también a nivel nacional y local, se hace necesaria la búsqueda de apropiación por el cuidado hacia el medio ambiente por parte de la niñez, ya que pese a los esfuerzos por conservar los recursos naturales no se ha podido frenar el deterioro ambiental (Fuentes, 2014). El aprendizaje del cuidado del medio ambiente debe iniciarse desde el currículo escolar y la vivencia de estrategias sencillas que desarrollen la conciencia ambiental en los futuros ciudadanos.

Los más recientes estudios demuestran que el deterioro ambiental no se limita únicamente en los países desarrollados, sino que la misma es alarmante en países subdesarrollados como Colombia. Entre las investigaciones realizadas en el área ambiental del país, se tiene el informe reportado por Duque (2019), donde se menciona que a pesar de que Colombia, es el segundo país latinoamericano en abundancia de agua y el segundo más biodiverso del mundo, solo se trata el $11 \%$ del agua utilizada y se ha deteriorado 27 tipos de ecosistemas de 85 identificados.

Entonces, ¿cómo enfrentar los conflictos socioambientales? para no dejarle a las siguientes generaciones, montañas deforestadas y erosionadas, y ríos contaminados y sedimentados, en un patrimonio natural cuya degradación se traduciría en desastres, ¿qué hacer para reducir pasivos ambientales?

Según Paz, Avendaño \& Parada (2014), esta problemática ambiental requiere de acciones concretas que promuevan el desarrollo sostenible haciendo uso adecuado de los recursos naturales, sin poner en peligro la existencia de las generaciones futuras. En este sentido, Cabezas y Barrios (2014) señalan cómo los organismos internacionales deben fortalecer el trabajo en educación ambiental en todos los sectores, ya que la conciencia ambiental no corresponde a un solo sector, sino que debe hacerse coordinadamente entre los diferentes sectores y miembros de una sociedad y/o comunidad, incluyendo el sector infantil.

La conciencia ambiental según Pita (2016), representa un proceso que le permite al individuo comprender las relaciones de interdependencia con el entorno en el que se 
desarrolla el cual tiene en cuenta la realidad biopsicosocial. Por otra parte, Bayon y Morejon (2005) señalan que la conciencia ambiental es un vínculo existente entre los individuos y su medio ambiente y que esta relación involucra tradiciones y situaciones a nivel social o colectivo, con características propias basada en su historia cultural.

Para lograr que la población a nivel mundial adquiera los conocimientos, aptitudes, actitudes y comportamientos necesarios ante los problemas actuales y prevenirlos en un futuro, se requiere trabajar en el contexto local, en este caso Cabezas y Barrios (2014), mencionan a las instituciones educativas como entes transformadores generadores de espacios y estrategias que lleven a los estudiantes a la realización de acciones locales. Por ello, el objetivo de esta investigación se centra en comprender la experiencia de los niños y niñas entre 4 y 5 años frente la responsabilidad ambiental en un colegio oficial de Tuluá, valle.

Debido a que el impacto ambiental de los últimos años ha desencadenado en la degradación de la calidad de vida de nuestra sociedad (Teófilo, 2019), se hace necesario desarrollar investigaciones que estudien la experiencia de los niños y niñas frente la responsabilidad ambiental. De otro lado, a partir de esta investigación se genera conocimientos para avanzar en nuevas estrategias de intervención que lleven a los menores a acciones contextualizadas acorde a sus necesidades y realidades de su localidad, lo cual permitirá desarrollar prácticas desde temprana edad que mitiguen el deterioro ambiental y que favorezcan la experiencia personal de los niños, respecto a la responsabilidad ambiental como ejercicio de conciencia, amor y protección hacia el medio ambiente.

En este orden, Guerra (2020) afirma que el constructivismo se ha convertido en paradigma tanto de planes educativos y programas de estudio y es conocido por su aplicación práctica gracias a los manuales de docencia, así como de la práctica de los docentes en gran parte de las instituciones educativas del mundo. González (2012), define el constructivismo como una posición psicológica y educativa que reúne varias teorías que coinciden en que los aprendizajes se construyen, no se transmiten, trasladan o se copian. Por lo cual, el constructivismo no es estrictamente una teoría, sino que es un marco explicativo, que parte de la consideración social e integra aportes de diversos autores. Entre sus grandes exponentes se encuentran las teorías de Jean Piaget, Lev Vygotsky, David Ausubel y Jerome Bruner. Aunque ninguno de ellos se denominó como constructivista, sus ideas y posturas claramente ilustran las ideas de esta corriente.

Vygotsky por su parte plantea que el niño tiene un conocimiento que le permite realizar determinadas tareas (zona de desarrollo) y el trabajo de los maestros, padres y cuidadores es trabajar la zona de desarrollo próximo, es decir, en las funciones que aún no han madurado en su totalidad, pero que se encuentran en proceso de maduración. Según Vygotsky (1979), los adultos o compañeros más avanzados deben ayudar a dirigir y organizar el aprendizaje del niño para que éste pueda internalizarlo, y esta guía es más eficaz para hacer que los niños crucen la zona de desarrollo próximo (ZDP), que es la brecha que hay entre lo que hacen y lo que todavía no logran por ellos mismos, pero que pueden lograr 
con la guía adecuada. Al respecto Papalia \& Martorell (2017) expresa que la responsabilidad de dirigir el aprendizaje pasa gradualmente al niño.

Domínguez (1997), describe que la corriente constructivista facilita convertir el aprendizaje tradicional en un aprendizaje innovador lo que implica en primer lugar trasformar un niño pasivo en uno activo; en segundo lugar, pasar de la planificación a la ejecución los principios constructivistas y de desarrollo de destrezas intelectuales superiores y finalmente atender el área socio-afectiva del menor, así como las variables contextuales donde se desarrolla el acto de aprender profundo. Ante ello Rosas \& Sebastián (2001), señalan 3 características esenciales de toda posición constructivista: primero, toda posición constructivista rescata al sujeto cognitivo. Segundo, a toda concepción constructivista en psicología le subyace, un determinado concepto de desarrollo, en este sentido se trata de explicar la "construcción" de ciertas estructuras a partir de otras que son diferentes. $Y$ tercero, toda posición constructivista tiene un interés por asuntos epistemológicos, por tanto, para el constructivismo es inevitable esclarecer preguntas ¿quién conoce?, ¿cómo conoce?, ¿qué conoce? y ¿qué es conocer?

\section{MÉTODO}

La investigación parte del paradigma cualitativo, el cual según Hernández \& Mendoza (2018) es un proceso inductivo contextualizado en un ambiente natural, esto se debe a que en la recolección de datos se establece una estrecha relación entre los investigadores y los participantes de la investigación de quienes se sustrae sus vivencias mediante el empleo de un instrumento de medición predeterminado (Flores, Gómez, \& Jiménez, 1999). Mediante el uso de esta metodología se podrá acceder a los testimonios de los niños y niñas de un colegio oficial de Tuluá, valle. Es de corte fenomenológico, ya "En el estudio, se pretende describir y entender los fenómenos desde el punto de vista de cada participante y desde la perspectiva construida colectivamente" (Hernández, Fernández, \& Baptista, 2006).

La muestra para esta investigación serán 11 niños de cuatro a cinco años, dentro de esta muestra se cuenta con 6 niños y 5 niñas. Por último, cabe mencionar que se contará con el consentimiento de sus cuidadores (padres o tutores responsables) y el de los mismos niños para el uso de la información la cual es con fines únicamente académicos. El método utilizado fue inductivo ya que parte de un contexto especifico y aportes teóricos que se intentan validar de manera empírica.

Los instrumentos utilizados fueron: entrevista a profundidad ya que permite indagar en los aspectos emocionales de los niños y niñas sobre sus conocimientos y actitud frente al cuidado del medio ambiente. Para Bautista (2011), la entrevista a profundidad es una técnica de investigación que permite obtener datos mediante un diálogo entre el investigador y el entrevistado. Por otra parte, se realizó una revisión documental, integrando la literatura existente relacionada con la conciencia ambiental y el desarrollo de los niños y niñas de en 
un colegio oficial de Tuluá, Valle. Esta búsqueda bibliográfica se realizó a través de bases de datos "Google scholar", "proquest", "PsycArticles", "ovid", "redalyc", "Ebsco", "Scielo".

a) El guion de la entrevista realizada fue:

\begin{tabular}{|c|c|c|}
\hline \multicolumn{3}{|c|}{ OBJETIVO GENERAL } \\
\hline \multicolumn{3}{|c|}{$\begin{array}{l}\text { Comprender la experiencia de los niños y niñas entre } 4 \text { y } 5 \text { años frente la responsabilidad ambiental } \\
\text { en un colegio oficial de Tuluá, valle. }\end{array}$} \\
\hline \multicolumn{3}{|c|}{ POBLACIÓN (MUESTRA) } \\
\hline \multicolumn{3}{|c|}{$\begin{array}{l}11 \text { niños de cuatro a cinco años matriculados en un nivel de educación preescolar de un colegio } \\
\text { oficial y que habiten en Tuluá valle. }\end{array}$} \\
\hline Temas (categorías) & Subtemas (subcategorías) & $\begin{array}{l}\text { Preguntas orientadoras (entrevista a } \\
\text { profundidad) }\end{array}$ \\
\hline \multirow[t]{3}{*}{ Medio Ambiente } & Entorno & ¿Sabes que es medio ambiente? \\
\hline & \multirow[t]{2}{*}{ Problema ambiental } & ¿Conoces algún problema que tenga el planeta? \\
\hline & & $\begin{array}{l}\text { ¿Me gustaría que me cuentes cuáles son los } \\
\text { recursos naturales que una vez se utilizan se } \\
\text { agotarán? }\end{array}$ \\
\hline \multirow[t]{3}{*}{$\begin{array}{l}\text { Responsabilidad } \\
\text { Ambiental }\end{array}$} & \multirow[t]{3}{*}{ Enfoque contextual social } & $\begin{array}{l}\text { ¿Cuándo tienes un papelito para botar, pero no } \\
\text { ves caneca que haces con él? }\end{array}$ \\
\hline & & $\begin{array}{l}\text { ¿Qué haces con el agua cuando te bañas los } \\
\text { dientes y las manos? }\end{array}$ \\
\hline & & ¿de qué manera cuidas las plantas? \\
\hline \multirow[t]{4}{*}{$\begin{array}{l}\text { Infancia y cuidado del } \\
\text { medio ambiente }\end{array}$} & Conciencia Ambiental & $\begin{array}{l}\text { ¿Cuál es el peligro que está corriendo el planeta } \\
\text { cuando las personas lo ensucian? }\end{array}$ \\
\hline & \multirow{3}{*}{$\begin{array}{l}\text { Educación ambiental y } \\
\text { constructivismo de Lev } \\
\text { Vygotsky }\end{array}$} & $\begin{array}{l}\text { ¿Sabes que cosas en el ambiente afectan los } \\
\text { seres vivos? }\end{array}$ \\
\hline & & ¿Para ti que es reciclar? \\
\hline & & $\begin{array}{l}\text { ¿Separas las botellas desechables, papeles y } \\
\text { plásticos? }\end{array}$ \\
\hline \multirow[t]{2}{*}{ Los niños de 4 a 5 años } & $\begin{array}{l}\text { Características del } \\
\text { desarrollo }\end{array}$ & $\begin{array}{l}\text { ¿Cómo te gustaría aprender a cuidar el medio } \\
\text { ambiente? }\end{array}$ \\
\hline & Reproducción cultural & $\begin{array}{l}\text { ¿de qué manera tus papitos o profes te enseñan } \\
\text { a cuidar el planeta (mundo, tierra)? }\end{array}$ \\
\hline \multirow[t]{2}{*}{ Información del contexto } & \multirow{2}{*}{$\begin{array}{l}\text { Población de infancia y } \\
\text { adolescencia en Tuluá } \\
\text { valle }\end{array}$} & $\begin{array}{l}\text { ¿Sientes que en tu ciudad las personas cuidan } \\
\text { el medio ambiente? }\end{array}$ \\
\hline & & $\begin{array}{l}\text { ¿Qué daño causan las personas al medio } \\
\text { ambiente? }\end{array}$ \\
\hline
\end{tabular}

Fuente: Elaboración propia.

Tabla 1. Resultados de la revisión documental se muestran a continuación:

\begin{tabular}{|l|l|l|l|l|l|l|l|l|}
\hline No. & AÑO & REFERENCIA & TITULO & LINK & BASE & OBJETIVOS & METODO & RESULTADOS \\
\hline
\end{tabular}




\begin{tabular}{|c|c|c|c|c|c|c|c|c|}
\hline 1. & 2014 & $\begin{array}{l}\text { Cabezas, M. R. } \\
\text { R., \& Barrios, E. } \\
\text { S. P. (2014). } \\
\text { Educación } \\
\text { ambiental en } \\
\text { niños y niñas de } \\
\text { instituciones } \\
\text { educativas } \\
\text { oficiales del } \\
\text { distrito de Santa } \\
\text { Marta. Zona } \\
\text { Próxima, (21), } \\
52-64 .\end{array}$ & $\begin{array}{l}\text { Educación } \\
\text { ambiental } \\
\text { en niños y } \\
\text { niñas de } \\
\text { instituciones } \\
\text { educativas } \\
\text { oficiales del } \\
\text { distrito de } \\
\text { Santa Marta }\end{array}$ & $\begin{array}{l}\text { https://www } \\
\text { redalyc.org } \\
\underline{\text { /pdf/853/85 }} \\
\underline{332835005} \\
\underline{\text {.pdf }}\end{array}$ & $\begin{array}{l}\text { Redaly } \\
\text { C }\end{array}$ & $\begin{array}{l}\text { Identificar el nivel } \\
\text { de conocimiento } \\
\text { de educación } \\
\text { ambiental en los } \\
\text { estudiantes de } \\
\text { primaria. }\end{array}$ & $\begin{array}{l}\text { enfoque } \\
\text { cuantitativo- } \\
\text { cualitativo, } \\
\text { descriptiva } \\
\text { y } \\
\text { transversal; }\end{array}$ & $\begin{array}{l}\text { La receptividad } \\
\text { encontrada en } \\
\text { los niños permite } \\
\text { inferir la } \\
\text { posibilidad de } \\
\text { aplicación } \\
\text { del concepto de } \\
\text { aprendizaje } \\
\text { significativo de } \\
\text { Ausubel y del } \\
\text { constructivismo } \\
\text { social de } \\
\text { Vigotsky para el } \\
\text { logro de } \\
\text { acciones } \\
\text { ambientales } \\
\text { contextualizadas } \\
\text { a sus realidades } \\
\text { y el } \\
\text { aprovechamient } \\
\text { o de sus } \\
\text { presaberes. }\end{array}$ \\
\hline 2. & 2014 & $\begin{array}{l}\text { Martínez, José } \\
\text { William } \\
\text { Desarrollo } \\
\text { infantil: una } \\
\text { revisión. } \\
\text { Investigaciones } \\
\text { Andinas. } \\
\text { 2014;16(29):11 } \\
\text { 18-1137.[fecha } \\
\text { de Consulta } 23 \\
\text { de Agosto de } \\
\text { 2020]. ISSN: } \\
\text { 0124-8146. }\end{array}$ & $\begin{array}{l}\text { Desarrollo } \\
\text { infantil: una } \\
\text { revisión. }\end{array}$ & $\begin{array}{l}\text { Disponible } \\
\text { en: } \\
\text { https://www } \\
\text { redalyc.org } \\
\text { larticulo.oa? } \\
\underline{\text { id=2390/23 }} \\
\underline{903167800} \\
\underline{9}\end{array}$ & $\begin{array}{l}\text { Redaly } \\
\text { C }\end{array}$ & $\begin{array}{l}\text { Explorar algunos } \\
\text { elementos } \\
\text { normativos y } \\
\text { conceptuales } \\
\text { que } \\
\text { fundamentan el } \\
\text { desarrollo } \\
\text { infantil. }\end{array}$ & $\begin{array}{l}\text { análisis } \\
\text { documental }\end{array}$ & $\begin{array}{l}\text { Se concluye que } \\
\text { la } \\
\text { agresión es un } \\
\text { evento que } \\
\text { incluye a las } \\
\text { mujeres con } \\
\text { maneras y } \\
\text { tendencias para } \\
\text { agredir }\end{array}$ \\
\hline 3. & 2019 & $\begin{array}{l}\text { Herrera-Mora, } \\
\text { D. B., Munar- } \\
\text { Torres, Y. E., } \\
\text { Molina-Achury, } \\
\text { N. J., \& Robayo- } \\
\text { Torres, A. L. } \\
\text { (2019). } \\
\text { Desarrollo }\end{array}$ & $\begin{array}{l}\text { Desarrollo } \\
\text { infantil y } \\
\text { condición } \\
\text { socioeconómic } \\
\text { a. Artículo de } \\
\text { revisión. }\end{array}$ & $\begin{array}{l}\text { https://web- } \\
\text { a- } \\
\text { ebscohost- } \\
\underline{\text { com.ibero.b }} \\
\underline{\text { asesdedato }} \\
\underline{\text { sezproxy.co }} \\
\underline{\text { m/ehost/pdf }} \\
\underline{\text { viewer/pdfvi }}\end{array}$ & Ebsco & $\begin{array}{l}\text { Conocer y } \\
\text { analizar la } \\
\text { influencia de las } \\
\text { condiciones } \\
\text { socioeconómicas } \\
\text { en el desarrollo } \\
\text { infantil. }\end{array}$ & $\begin{array}{l}\text { análisis } \\
\text { documental }\end{array}$ & $\begin{array}{l}\text { Se encontró una } \\
\text { relación } \\
\text { significativa } \\
\text { entre } \\
\text { desarrollo infantil } \\
\text { y nivel } \\
\text { socioeconómico, } \\
\text { escolaridad, }\end{array}$ \\
\hline
\end{tabular}




\begin{tabular}{|c|c|c|c|c|c|c|c|c|}
\hline & & $\begin{array}{l}\text { infantil y } \\
\text { condición } \\
\text { socioeconómica } \\
\text {. Artículo de } \\
\text { revisión. Revista } \\
\text { de la Facultad } \\
\text { de Medicina, } \\
67(1), 145-152 .\end{array}$ & & $\begin{array}{l}\text { ewer?vid=1 } \\
\text { \&sid=87568 } \\
\underline{053-e e 24-} \\
\underline{4186-a 0 a c-} \\
\text { a7847b9b4 } \\
\underline{402 \% 40 s d c} \\
\underline{\text {-v- }} \\
\underline{\text { sessmgr03 }}\end{array}$ & & & & $\begin{array}{l}\text { ocupación de los } \\
\text { padres, estado } \\
\text { civil de la madre } \\
\text { y condiciones de } \\
\text { la vivienda, que } \\
\text { suponen } \\
\text { una desventaja y } \\
\text { la probabilidad } \\
\text { de alteración en } \\
\text { el desarrollo } \\
\text { infantil. }\end{array}$ \\
\hline 4. & 2015 & $\begin{array}{l}\text { Souza, J. M. D., } \\
\text { \& Veríssimo, M. } \\
\text { D. L. Ó. R. } \\
\text { (2015). } \\
\text { Desarrollo } \\
\text { infantil: análisis } \\
\text { de un nuevo } \\
\text { concepto. } \\
\text { Revista latino- } \\
\text { americana de } \\
\text { enfermagem, } \\
\text { 23(6), 1097- } \\
\text { 1104. }\end{array}$ & $\begin{array}{l}\text { Desarrollo } \\
\text { infantil: análisis } \\
\text { de un nuevo } \\
\text { concepto }\end{array}$ & $\begin{array}{l}\frac{\text { https://www }}{\text { scielo.br/p }} \\
\underline{\text { df/rlae/v23n }} \\
\underline{\text { 6/es_0104- }} \\
\underline{\text { 1169-rlae- }} \\
\underline{\text { 23-06- }} \\
\underline{\text { 01097.pdf }}\end{array}$ & Scielo & $\begin{array}{l}\text { Realizar el } \\
\text { análisis del } \\
\text { concepto } \\
\text { "desarrollo } \\
\text { infantil” y } \\
\text { evaluarlo con } \\
\text { expertos. }\end{array}$ & $\begin{array}{l}\text { Investigació } \\
\text { n } \\
\text { cualitativa, } \\
\text { se utilizó el } \\
\text { modelo } \\
\text { híbrido } \\
\text { de } \\
\text { desarrollo } \\
\text { de } \\
\text { conceptos. }\end{array}$ & $\begin{array}{l}\text { Se generó una } \\
\text { nueva definición } \\
\text { para las } \\
\text { clasificaciones } \\
\text { de enfermería y } \\
\text { la atención } \\
\text { integral a los } \\
\text { niños }\end{array}$ \\
\hline 5. & 2014 & $\begin{array}{l}\text { Paz, L. S., } \\
\text { Avendaño, W. } \\
\text { R., \& Parada- } \\
\text { Trujillo, A. E. } \\
\text { (2014). } \\
\text { Desarrollo } \\
\text { conceptual de la } \\
\text { educación } \\
\text { ambiental en el } \\
\text { contexto } \\
\text { colombiano. Rev } \\
\text { ista luna azúl, } \\
\text { (39), 250-270. }\end{array}$ & $\begin{array}{l}\text { DESARROLLO } \\
\text { CONCEPTUAL } \\
\text { DE LA } \\
\text { EDUCACIÓN } \\
\text { AMBIENTAL } \\
\text { EN EL } \\
\text { CONTEXTO } \\
\text { COLOMBIANO }\end{array}$ & $\begin{array}{l}\text { https://www } \\
\text { redalyc.org } \\
\text { /pdf/3217/3 } \\
\underline{217321420} \\
\underline{15 . p d f}\end{array}$ & $\begin{array}{l}\text { Redaly } \\
\text { C }\end{array}$ & $\begin{array}{l}\text { Explorar algunos } \\
\text { elementos } \\
\text { normativos y } \\
\text { conceptuales } \\
\text { que } \\
\text { fundamentan la } \\
\text { educación } \\
\text { ambiental, así } \\
\text { como la } \\
\text { adopción de la } \\
\text { misma en } \\
\text { términos } \\
\text { curriculares en } \\
\text { Colombia. }\end{array}$ & $\begin{array}{l}\text { análisis } \\
\text { documental }\end{array}$ & $\begin{array}{l}\text { La educación } \\
\text { ambiental ha } \\
\text { sido asumida } \\
\text { conforme a las } \\
\text { características } \\
\text { de cada periodo } \\
\text { histórico con un } \\
\text { gran cúmulo de } \\
\text { normas } \\
\text { internacionales y } \\
\text { nacionales, y } \\
\text { que su } \\
\text { implementación } \\
\text { desde la escuela } \\
\text { debe } \\
\text { corresponder a } \\
\text { una reflexión } \\
\text { conjunta y } \\
\text { colectiva de }\end{array}$ \\
\hline
\end{tabular}




\begin{tabular}{|c|c|c|c|c|c|c|c|c|}
\hline & & & & & & & & $\begin{array}{l}\text { todos los } \\
\text { actores } \\
\text { escolares, tanto } \\
\text { en su diseño } \\
\text { como } \\
\text { implementación. }\end{array}$ \\
\hline 6. & 2014 & $\begin{array}{l}\text { Souza, J. M. D. } \\
\text { (2014). } \\
\text { Desenvolviment } \\
\text { o infantil: análise } \\
\text { de conceito e } \\
\text { revisão dos } \\
\text { diagnósticos da } \\
\text { NANDA-I } \\
\text { (Doctoral } \\
\text { dissertation, } \\
\text { Universidade de } \\
\text { São Paulo). }\end{array}$ & $\begin{array}{l}\text { Desarrollo } \\
\text { infantil: análisis } \\
\text { de conceptos y } \\
\text { revisión de } \\
\text { diagnósticos } \\
\text { NANDA-I }\end{array}$ & $\begin{array}{l}\text { https://www } \\
\text { teses.usp.b } \\
\frac{\text { r/teses/disp }}{\text { oniveis/7/71 }} \\
\underline{41 / \text { tde- }} \\
\underline{05112014-} \\
\underline{115040 / p u b} \\
\text { lico/DOUTO } \\
\underline{\text { RADO_JULI }} \\
\text { ANA_MART } \\
\text { INS_DE_SO } \\
\text { UZA.pdf }\end{array}$ & $\begin{array}{l}\text { Bibliot } \\
\text { eca } \\
\text { Digital } \\
\text { de } \\
\text { Tesis y } \\
\text { Disert } \\
\text { acione } \\
\text { s de la } \\
\text { USP. }\end{array}$ & $\begin{array}{l}\text { Abordar el } \\
\text { desarrollo infantil } \\
\text { de manera } \\
\text { integral en el } \\
\text { proceso de } \\
\text { enfermería. }\end{array}$ & $\begin{array}{l}\text { Investigació } \\
\mathrm{n} \\
\text { cualitativa. } \\
\text { Análisis } \\
\text { documental }\end{array}$ & $\begin{array}{l}\text { El análisis de } \\
\text { concepto } \\
\text { proporcionó una } \\
\text { nueva definición } \\
\text { de EC que } \\
\text { apoyó nuevos } \\
\text { diagnósticos } \\
\text { para la mejora } \\
\text { de la práctica de } \\
\text { enfermería. }\end{array}$ \\
\hline
\end{tabular}

Fuente: Elaboración propia.

\section{RESULTADOS Y DISCUSIÓN}

Los resultados obtenidos muestran que existe una relación directa entre la conciencia ambiental y la construcción cultural que el niño hace diariamente al interactuar con sus maestros y cuidadores principales. Lo cual ratifica la validez de la teoría de Vigotsky al señalar que el aprendizaje es un proceso colaborativo de participación guiada, el cual hace referencia a aquellas interacciones del niño con los adultos que ayudan a estructurar las acciones de los infantes y que ocurren mediante el juego compartido y en actividades cotidianas ya que de este modo aprenden de manera informal habilidades, conocimientos y valores importantes de su cultura (Papalia \& Martorell, 2017).

Tabla 2. Los niños de 4 a 5 años

\section{Características del desarrollo}

\section{- Percepción de acciones sociales: colaboración} (enseñanza por parte de la madre)

- Expresiones culturales: iniciativa de aprendizaje (que no sea aburrido, Jugando con mis amigos en el colegio)
Los niños de 4 a 5 años

Reproducción
cultural

- Expresiones interacción con los cuidadores (me enseñan botar la basura en la caneca y a cuidar los animales)

- Expresiones culturales: (con los amigos) 
Fuente: elaboración propia

La experiencia descrita por los niños señala como estos hacen una construcción propia de sus aprendizajes producto de las vivencias personales acompañada de la información proveniente principalmente de sus cuidadores y maestros. Tras comparar estos hallazgos con el sustento teórico de nuestro trabajo, se encuentra convergencia con el mismo, ya que teniendo en cuenta la teoría de Lev Vygotsky (1989) que afirma que el crecimiento del niño se da tanto dentro de la sociedad fusionado con los procesos de maduración orgánica (Werstch y Vygotsky, 1988) en el cual ambos procesos (el proceso del desarrollo natural y el cultural) se fusionan entre sí y los resultados encontrados en la presente investigación convergen ante esta afirmación teórica, puesto que las expresiones culturales (hábitos, costumbres y normas) impartidas por los cuidadores y maestros son expresadas por el niño como parte del significado que el niño da sobre la responsabilidad ambiental, mostrando así la interiorización de los nuevos aprendizajes, ya que, como menciona Coll (1990) el verdadero artífice del proceso de aprendizaje es el alumno.

Tabla 3. Información del contexto

$\begin{array}{cll} & \text { Población de } & \text { Percepción de riesgo ambiental: } \\ \text { infancia y } & \text { expresiones de daño } \\ \text { adolescencia } & \text { Ambiental } \\ \text { del contexto } & \text { (Las personas riegan basura en las } \\ & \text { calles) } \\ & \text { Los adultos matan los animalitos y los } \\ & \text { arboles } \\ & \text { estados de asimilación: expresión de } \\ \text { cuidado } & \end{array}$

\begin{tabular}{|c|c|c|}
\hline & Entorno & $\begin{array}{l}\text { - Malestar personal: evasión de problemas } \\
\text { (Si, este mmm no sé) } \\
\text { - } \quad \text { Estado de asimilación: bienestar } \\
\text { (es un lugar que debemos cuidar) }\end{array}$ \\
\hline Medio Ambiente & $\begin{array}{l}\text { Problema } \\
\text { ambiental }\end{array}$ & $\begin{array}{ll}\text { - } & \text { Percepción de riesgo ambiental: } \\
& \text { expresiones de daño ambiental } \\
\text { - } & \text { (Si acá el parque, botan basura) } \\
\text { - } & \text { Malestar personal: confirmación negativa } \\
\text { - } & \text { (Yo una vez estaba en el parque y un niño } \\
& \text { arrancó las flores que había) } \\
\text { - } & \text { Percepción de acciones sociales: } \\
& \text { expresiones de contaminación } \\
\text { - el agua es si se ensucia ya no se puede } \\
\text { limpiar) }\end{array}$ \\
\hline
\end{tabular}

Fuente: elaboración propia

Desde la fenomenología cabe señalar que la experiencia para los niños entrevistados da cuenta que a pesar de que el macro sistema es indicado como una fuente de malestar personal en la cual los niños manifiestan expresiones de desaprobación ante ciertas conductas realizadas por sus vecinos, familia extendida y miembros de su comunidad, estos 
no configuran una apropiación ni internalización de dichos patrones comportamentales, ya que son los padres y cuidadores de quienes internalizan los modos de pensar y actuar de su sociedad y en este caso sobre la responsabilidad ambiental. Es por ello que Vygotsky (1979), sostiene "el aprendizaje humano presupone una naturaleza social específica y un proceso, mediante el cual los niños acceden a la vida intelectual de aquellos que los rodean" (p.136), resaltando la importancia de los adultos en el proceso de aprendizaje y su trascendencia para trasmitir el proceso cultural del aprendizaje, de allí que, se deben generar cambios en la instrucción ambiental impartida en infantes desde temprana edad que genere cambios positivos en su percepción hacia la cultura ambiental por medio de la búsqueda de estrategias de enseñanza (Epiquén, Quispe, \& Verde, 2020).

Tabla 4. Responsabilidad Ambiental

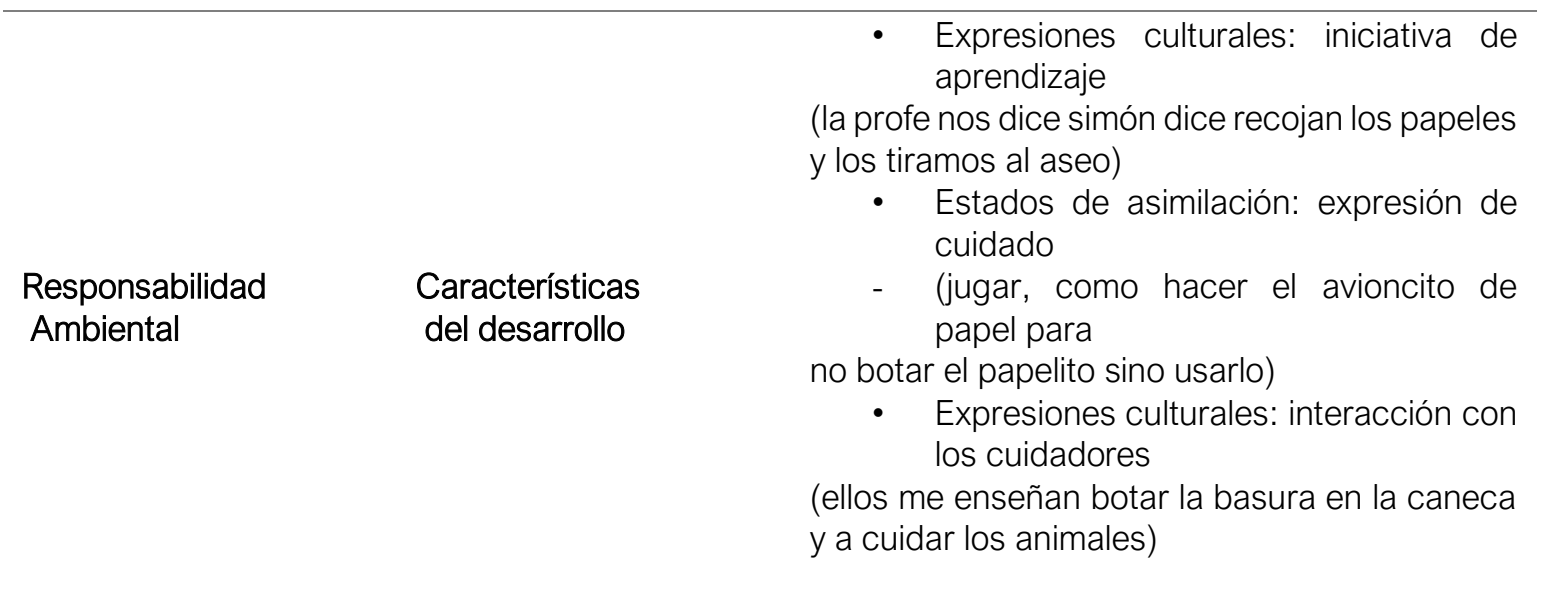

Fuente: elaboración propia

En este estudio se encontró que el entorno inmediato (padres y cuidadores) constituyen la principal fuente de gestión de conocimiento para el desarrollo de una conciencia ambiental. Según Ledesma (2014), la mediación tiene como objetivo construir con una persona esquemas para potenciar sus capacidades y en el ámbito de educación ambiental que es fundamental llevar a cabo el andamiaje aplicado, donde la "ayuda del mediador" para su aprendizaje dará como resultado la resolución de problemas enfocado en la adquisición de una conciencia ambiental, que garantice según Flores (2012) una convivencia en armonía y paz entre ser humano y medio ambiente, ya que "la falta de conciencia ambiental genera actitudes que ocasionan daño al medio ambiente" (Cerón, Delgado \& Benavides, 2015, p. $44)$, por lo cual se deben generar prácticas ambientales que propicien el desarrollo de la conciencia ambiental.

Tabla 5. Infancia y cuidado del medio ambiente

\begin{tabular}{lll} 
Conciencia & $\bullet$ & $\begin{array}{l}\text { Percepción de riesgo ambiental: } \\
\text { expresiones de contaminación } \\
\text { Ambiental } \\
\text { (Pues que el agua se pone negra y no } \\
\text { se puede tomar porque está sucia) }\end{array}$ \\
\hline
\end{tabular}


- Expresiones culturales: interacción con los cuidadores

Infancia y cuidado del medio ambiente
- (mi mamá me enseño que planta que le cae basura se daña la planta)

\begin{tabular}{l}
\hline Educación \\
ambiental y \\
constructivismo \\
de \\
Lev Vygotsky
\end{tabular}

- Expresiones culturales: interacción cuidadores

- (mi mama me enseñó a que toca cerrar la llave)

- Percepción de acciones sociales: iniciativa de cambio

- (echar la basura en canecas de colores.)

Fuente: elaboración propia

De acuerdo con la entrevista desarrollada, se encontró que la conciencia ambiental de los niños y niñas está relacionada principalmente con el cuidado del agua, el cuidado de las plantas y no arrojar basura al suelo. No obstante, se observa dificultad para que los menores puedan realizar la transición de la zona del desarrollo real a la zona de desarrollo próximo relacionada con el manejo de contenedores clasificadores de los residuos sólidos, esto probablemente debido a que los niños aún no han llegado a su zona de desarrollo próximo como lo menciona Vygotsky (1979), es decir, que este concepto aún no ha madurado en su totalidad, pero que se encuentran en proceso de maduración, (Puche, Orozco, Orozco, \& Correa, 2009) también reconocen que existen ciertas irregularidades en el acceso al conocimiento y al manejo de la forma que se toma el desarrollo y de cómo funcionan la mente y la afectividad de los niños.

De otro lado Gutiérrez (2015), menciona que se habla mucho de los daños ambientales, pero no existe una reflexión ni el desarrollo de actitudes y valores ecológicos que pretendan mejorar la situación, de allí la importancia de articular modelos de aprendizaje que permitan un mayor énfasis en el desarrollo de una conciencia ambiental, en tal caso se tiene en cuenta que, a través de los años, se desarrollaron tres teorías clásicas: el conductismo, el cognitivismo y el constructivismo (Cejudo y Almenara, 2015). Cada una, presenta ventajas y desventajas, lo que ha llevado a no tomar ninguna teoría como absoluta, sino a buscar una especialización y desarrollo en una teoría especifica según las características docentes y su desarrollo académico (Pérez, Miguelena y Diallo, 2016). La comprensión y aplicación de cada teoría habilita al personal docente para alcanzar una mejor transposición pedagógica con el estudiantado, de acuerdo con sus características y capacidades, para generar un mejor ambiente de aprendizaje en el proceso. 
Tabla 5. Medio Ambiente

\begin{tabular}{|c|c|c|}
\hline & Entorno & $\begin{array}{l}\text { - Malestar personal: evasión de } \\
\text { problemas } \\
\text { (Si, este mmm no sé) } \\
\text { - Estado de asimilación: bienestar } \\
\text { (es un lugar que debemos cuidar) }\end{array}$ \\
\hline Medio Ambiente & $\begin{array}{l}\text { Problema } \\
\text { ambiental }\end{array}$ & 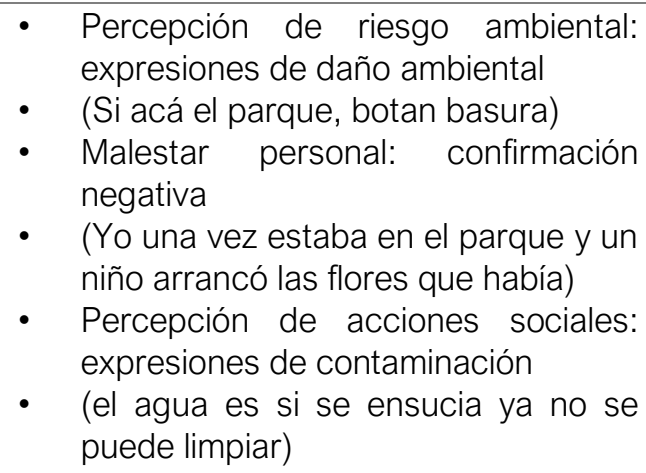 \\
\hline
\end{tabular}

Fuente: elaboración propia

Finalmente, este estudio encontró que la ausencia de modelos de aprendizaje sobre responsabilidad ambiental en niños de 4 y 5 años en un colegio oficial de Tuluá, Valle responde a la necesidad de realizar un mejor andamiaje en los menores que este mediado por el juego y la conexión emocional, no obstante, los niños entre 4 y 5 años han demostrado que a pesar de las escasas estrategias educativas para el desarrollo de una conciencia ambiental efectiva en esta área, son precisamente los niños la principal potencia para generar el cambio a nivel mundial, ya que sus experiencias presentan un nivel más elevado de conciencia hacia la defensa de los ecosistemas y la naturaleza.

Según el Fondo de las Naciones Unidas para la Infancia UNICEF (2008), los niños son más críticos y receptivos que los adultos ante las situaciones ambientales, por lo que la formación ambiental enfocada a este grupo poblacional a través de unos adecuados modelos de aprendizaje desde épocas tempranas del desarrollo sobre responsabilidad ambiental puede ayudar a generar nuevos recursos a los problemas ambientales, y también permitirá que cada niño sea un futuro embajador del cambio en la conducta ambiental de su cultura.

\section{CONCLUSIONES}

Con respecto al objetivo general se encontró que, durante su infancia, los niños adquieren diferentes capacidades a nivel biopsicosocial, entre ellas el desarrollo de su conciencia ambiental. En este sentido, los niños participantes tienen la experiencia respecto a la responsabilidad ambiental asociada al cuidado de las plantas, el agua y el manejo de los residuos sólidos. Este proceso de conciencia ambiental fue adquirido a través de la interrelación con las demás personas, y en esta investigación, específicamente con los 
cuidadores principales y maestros y es así como han construido la estructura para la apropiación de la conciencia ambiental, el reconocimiento de su rol y el de los otros respecto a la responsabilidad ambiental en Tuluá, Valle.

La receptividad encontrada en los niños y niñas de la institución educativa oficial en Tuluá, Valle permite la aplicación del constructivismo social de Vygotsky para el logro de acciones ambientales contextualizadas a sus realidades y el aprovechamiento de sus pre saberes, para obtener un alto nivel de motivación sobre la apropiación de nuevos conocimientos para el manejo del entorno ambiental. También se pudo identificar actitudes de cuidado y protección del medio ambiente pues manifiestan sentir malestar personal frente a las actitudes de los adultos cuando tiran al piso su basura, ya que consideran que esta es una mala acción, manejan en sus expresiones de daño al medio ambiente, conceptos muy claros frente a la importancia de cuidar el agua, dar el debido tratamiento final a los residuos y proteger y cuidar las plantas.

Se evidenció que para mejorar la percepción ambiental en niños es necesaria una reorientación hacia la formación en modelos de aprendizaje ambiental en el hogar y los entornos educativos. Por lo cual, se sugiere a los docentes la implementación de modelos de aprendizaje ambiental que mejoren la percepción ambiental en niños y niñas. Finalmente, y para futuras investigaciones, se recomienda utilizar preguntas más específicas teniendo en cuenta el contexto y situación actual en la que el niño se desenvuelve. En este sentido, nos encontramos con muchas respuestas relacionadas en el marco del COVID 19 ante preguntas relacionadas con el medio ambiente ya que para los niños en el "ambiente" está el virus.

\section{REFERENCIAS}

Álvarez. A. (1997). Hacía un currículum cultural. La vigencia de Vigotsky en la educación. Madrid: Editorial Fundación Infancia y Aprendizaje.

Avendaño, W. \& Parada, A. (2011). Un modelo pedagógico para la reproducción y transformación cultural en las sociedades del conocimiento. Investigación y Desarrollo, 19(2), 398-413.

Avendaño, D. (2014). El derecho a un ambiente sano y los derechos humanos: Aproximaciones para una reparación integral del daño ambiental, Revista Global lure del daño ambiental. Universidad Estatal a Distancia de Costa Rica. (No. 002872 TFG).

Avendaño, W. (2011). Responsabilidad Social Corporativa (RSC) y desarrollo sostenible: una mirada desde la Declaración de Río de 1992. Respuestas, 16(2), 45-59.

Briolotti, A. (2015). Desarrollo psicológico, naturaleza y cultura en la teoría de Arnold Gesell. Memorandum: Memória e História em Psicologia, 28, 55-70.

Bautista, C. (2011). Proceso de la investigación cualitativa: Epistemología, metodología y aplicaciones. Manual Moderno.

Bayon, P. y Morejón, A. (2005). Cultura ambiental y construcción de entornos de producción 
social en Cuba: Un reto para el siglo 21. Instituto de Filosofía; Ministerio de ciencia Tecnología y medio Ambiente.

Cabezas, M., \& Barrios, E. (2014). Educación ambiental en niños y niñas de instituciones educativas oficiales del distrito de Santa Marta. Zona Próxima, (21), 52-64.

Carrera, B., \& Mazzarella, C. (2001). Vygotsky: enfoque sociocultural. Educere, 5(13), 41 44.

Cejudo, M. y Almenara, J. (2015). Tecnologías de la información y la comunicación (TIC): Escenarios formativos y teorías del aprendizaje. Revista Lasallista de Investigación, 12(2), 25-36.

Chica, M., \& Prado, A. (2012). La construcción social de la infancia y el reconocimiento de sus competencias. Itinerario Educativo, 26(60), 75-96.

Cerón, A., Delgado, G. \& Benavides, E. (2015). Desarrollo de valores ambientales a través de una didáctica creativa (Tesis de especialidad).

Coll, C., \& Gillièron, C. (1981). Jean Piaget y la Escuela de Ginebra: itinerario y tendencias actuales. Infancia y Aprendizaje, 4(sup2), 56-96.

Coll, C. (1990). Un marco de referencia psicológico para la educación escolar: la concepción constructivista del aprendizaje y la enseñanza. En: C. Coll, J. Chomsky, N. (1980). El lenguaje y el conocimiento inconsciente. México: FCE.

Colmenares, A. (2012). Investigación-acción participativa: Una metodología integradora del conocimiento y la acción. Voces y Silencios: Revista Latinoamericana de Educación, 3(1), 102-115.

C. P. (1991). Constitución política de Colombia. Bogotá, Colombia: Leyer.

Dewey, J. (2004). Democracia y educación. Madrid: Morata.

Domínguez, M. (1997) Efectos de Pantalla y Constructivismo. En: Pensamiento Vol. XXI, diciembre, Santiago de Chile.

Duque, G. (2019). Un país con grandes retos ambientales. Observatorio Astronómico de Manizales (OAM) [138], Universidad Nacional de Colombia, Caldas, Manizales.

Epiquén, A., Quispe, J. \& Verde, M. (2020). Estudio comparativo sobre la Cultura ambiental en niños y niñas de 5 años de Huancayo, Perú. Telos: Revista de Estudios Interdisciplinarios en Ciencias Sociales, 22(3), 528-540.

Estrada, R., Monroy, G. \& Ramírez, H. (2005). Ética-responsabilidad-social- desarrollo sustentable en las organizaciones. Administración y Organizaciones, 8(15), 27-45.

Estrada, L. (2017). Desarrollo y ciclo vital: niños y adolescentes (Primera ed.). Bogotá: Fundación Universitaria del Área Andina.Fuentes Olmos, J. (2014). Evolución del régimen ambiental de la acuicultura en Chile. Revista de derecho (Valparaíso), (42), 441-477.

Fuentes, J. (2014). Evolución del régimen ambiental de la acuicultura en Chile. Revista de derecho (Valparaíso), (42), 441-477.

Flores, J., Gómez, G., \& Jiménez, E. (1999). Metodología de la investigación cualitativa. Málaga: aljibe.

Flores, R. (2012). Investigación en educación ambiental. Revista mexicana de investigación educativa, 17(55), 1019-1033. 
Flórez-Yepes, G. (2015). La educación ambiental y el desarrollo sostenible en el contexto colombiano. Revista Electrónica Educare, 19(3), 432-443.

Formación ambiental (2002) Órgano Informativo de la Red de Formación Ambiental para América Latina y el Caribe. México, volumen 14, núm. 30.

Giroux, H. (1986). Teorías de la reproducción y la resistencia en la nueva sociología de la educación: un análisis crítico. Revista Colombiana de Educación, 17, 61-108.

Göncü, A., Mistry, J., \& Mosier, C. (2000). Cultural variations in the play of toddlers. International Journal of Behavioral Development, 24(3), 321-329.

González, C. (2012). Aplicación del constructivismo social en el aula.

Gutiérrez, L. (2015). Problemática de la educación ambiental en las instituciones educativas. Revista Científica, 57-76.

Hernández-Sampieri, R., \& Torres, C. (2018). Metodología de la investigación (Vol. 4). México^ eD. FDF: McGraw-Hill Interamericana.

Hernández Sampieri, R., Fernández Collado, C. y Baptista Lucio, P. (2006). Metodología de la investigación. 4 a ed.

Herrera-Mora, D., Munar-Torres, Y., Molina-Achury, N., \& Robayo-Torres, A. (2019). Desarrollo infantil y condición socioeconómica. Artículo de revisión. Revista de la Facultad de Medicina, 67(1), 145-152.

Holguín, M., Bonilla, P. E., Pupo, A., Lezaca, J., Rodríguez, I., \& Rodríguez, T. (2013). Guía metodológica para la formulación de proyectos ambientales escolares. Un reto más allá de la escuela. Bogotá: Universidad Libre/Jardín Botánico de Bogotá José Celestino Mutis.

Jiménez, M., \& Lafuente, R. (2010). Definición y medición de la Conciencia Ambiental. Revista Internacional de Sociología, 68(3), 731-755.

Leff, E. (2000). Pensar la complejidad ambiental. La complejidad ambiental, 7-53.

Ledesma, M. (2014). Análisis de la teoría de Vygotsky para la reconstrucción de la inteligencia social. Cuenca, Ecuador: Universidad Católica de Cuenca. Recuperado de https://gredos. usal. es/jspui/bitstream/10366/127738/1/LIBRO-VYGOTSKY. pdf.

McMillan, J., Schumacher, S., \& Baides, J. (2005). Investigación educativa: una introducción conceptual. Madrid: Pearson.Marín, G. P. (1988). Consideraciones acerca del estado actual de la educación física en Colombia. Educación Física y Deporte, 10(1-2), 8594.

Martínez, S. (2019). Pensamiento ambiental latinoamericano y protagonismo docente en escuelas públicas. Revista nuestrAmérica, 7(14), 55-70.

Martínez, J. (2014) Desarrollo infantil: una revisión. Investigaciones Andina. 2014;16(29):1118-1137. [Fecha de Consulta 23 de Agosto de 2020]. ISSN: 01248146.

Muñoz-Cadena, C., Estrada-Izquierdo, I. \& Morales-Pérez, R. (2016). Logros de la educación ambiental y la sustentabilidad urbana en México. Revista electrónica de investigación educativa, 18(3), 37-50.

Moshman, D. (1982) Constructivismo Endógeno, Exógeno y Dialéctico. Developmental Review 2, 371- 384. 
Organización de las Naciones Unidas (1997)., Comisión de Derechos Humanos, 49vo periodo de sesiones. Informe final revisado acerca de la cuestión de la impunidad de los autores de violaciones de los derechos humanos (derechos

Otzen, T., \& Manterola, C. (2017) Técnicas de Muestreo sobre una Población a Estudio, Rev. Scielo, $35 \quad$ (1) Pp. 227-232, disponible en: https://scielo.conicyt.cl/pdf/ijmorphol//35n1/art37.pdf

Papalia, D., \& Martorell, G. (2017). Desarrollo Humano (decimotercera edición ed.). Mexio: McGRAW-HILL/INTERAMERICANA EDITORES, S.A. DE C.V.

Paz, L., Avendaño, W., \& Parada-Trujillo, A. (2014). Desarrollo conceptual de la educación ambiental en el contexto colombiano. Revista luna azúl, (39), 250-270.

Pérez, L., Miguelena, R., \& Diallo, A. (2016). La efectividad de la formación en ambientes virtuales de aprendizaje en la educación superior. Campus Virtuales, 5(2), 10-17.

Pita-Morales, L. (2016). Línea de tiempo: educación ambiental en Colombia. Praxis, 12(1), 118-125.

Puche, R., Orozco, H., Orozco, B., \& Correa, M. (2009). Desarrollo infantil y competencias en la primera infancia. Ministerio de educación nacional. Bogotá, Colombia: Colombia aprende.

Referente Curricular de educación inicial, "Currículo de Nivel Inicial". Ecuador - Quito, Editorial Ministerio de Educación (2013).

Rengifo, B., Quitiaquez, L., \& Mora, F. (2012). La educación ambiental una estrategia pedagógica que contribuye a la solución de la problemática ambiental en Colombia. XII Coloquio internacional de Geocrítica, 16.

Robles, B. (2011). La entrevista en profundidad: una técnica útil dentro del campo antropofísico. Cuicuilco, 18(52), 39-49.

Rojas, O., \& Londoño, A. (2016). De la educación ambiental hacia la configuración de redes de sostenibilidad en Colombia. Perfiles educativos, 38(151), 175-187.

Rosas, R., \& Sebastián, C. (2001). Piaget, Vigotski y Maturana: Constructivismo a tres voces (pp. 8-9). Buenos Aires: Aique.

Schmitt, J., Neale, M., Clasen, L., Liu, S., Seidlitz, J., Pritikin, J., \& Raznahan, A. (2019). Un análisis genético cuantitativo integral de la superficie cerebral en la juventud. Revista de neurociencia, 39 (16), 3028-3040.

Sierra, C., Morales, J. y Barrios, R. (2014). Mapas conceptuales como estrategia de enseñanza-aprendizaje en las ciencias ambientales. Itinerario Educativo, 28(64), 163-176.

Souza, J. (2014). Desenvolvimento infantil: análise de conceito e revisão dos diagnósticos da NANDA-I (Doctoral dissertation, Universidade de São Paulo).

Souza, J., \& Veríssimo, M. (2015). Desarrollo infantil: análisis de un nuevo concepto. Revista latino-americana de enfermagem, 23(6), 1097-1104.

Teófilo-Salvador, E., Morales-Reyes, G., Muciño-Castañeda, R., \& Esteller-Alberich, M. (2019). Experimentación reducida-controlada in situ del deslizamiento de suelo por efecto de flujo subsuperficial de agua. Ingeniería, investigación y tecnología, 20(3).

Torrenteras, J. (2015). Las teorías de aprendizaje y la formación de herramientas técnicas. 
Revista de Educación a Distancia, 34, 25-31.

Tyler, R. (1986). Principios básicos del currículo. Buenos Aires: Troquel.

Valdés, O., Rodríguez, A., Llivina, M., Betancourt, A. \& Santos, I. (2012). La educación ambiental y desarrollo sostenible: Estrategias de integración interdisciplinaria curricular e institucional en los programas, proyectos y buenas prácticas en las universidades, escuelas, familias y comunidades en Cuba. Ministerio de Educación. La Habana: Educación Cubana.

Vygotsky, L.S. (1979). Pensamiento y lenguaje. Buenos Aires: La pléyade.

Werstch, J. \& Vigotsky (1988). La formación social de la mente. Barcelona: Paidós 